\title{
A generalization of Bochner's formula
}

\author{
S. Kanemitsu, Y. Tanigawa and H. Tsukada \\ Dedicated to Professor Yoshinobu Nakai on his sixtieth birthday
}

\begin{abstract}
In this note we expound our general hierarchy theorems by the example of a Ramified-Type Functional Equarion H, which gives all possbile forms, in terms of series with $\mathrm{H}$-function coefficients, of the functional equation of higher hierarchy arising from the original ramified one satisfied by the Dirichlet series. Then by sepcifying the parameters, we shall deduce a few concrete examples scattered in the literature in the most natural way.
\end{abstract}

\section{Introduction}

S. Bochner, developing the theory of E. Hecke, opened a new entrance to the land of zetafunctions satisfying the functional equation with multiple Gamma factors, i. e. the modular relation principle ([2], [3]). We have been trying to interpret the existing formulas of zetafunctions in the framework of the modular relation, with a diversity of results culminating in [14]. A breakthrough in research was made by the third author, who, under the suggestion of the first author to utilize generalized hypergeometric functions, has considered the problem in an even more general setting, i. e. in the realm of the Meijer G-functions and the Fox $\mathrm{H}$-functions, resulting in a tremendous success, as is reported in [18] and being put into the book form [13].

In this note, we shall state a special case of our Main Formula $\mathrm{H}$ as the Ramified Type Main Formula and illustrate it by some concrete examples which are of interest in their own right. In section 2 , we shall give yet another proof of the functional equation for the Hurwitz-Lerch zeta-function

$$
\phi(z, s, a)=\sum_{k=0}^{\infty} \frac{e^{2 \pi i k z}}{(k+a)^{s}} \quad(0<a \leq 1)
$$

in addition to other proofs given in [11] as a Hecke-type zeta-function. Our present method assumes the $\mathrm{L} \rightarrow \mathrm{H}$ formula and deduces the incomplete gamma series (2.17), from which the functional equation may be deduced in various ways. In section 3 , we shall take up the result of Johnson [8] relating to the functional equation for the generalized Hurwitz-Lerch zeta-function

$$
\Phi_{A}(z, s, a)=\sum_{k=0}^{\infty} \frac{e^{-z^{A}(k+a)^{A}}}{(k+a)^{s}} \quad(0<a \leq 1, A>0)
$$

Note that

$$
\phi(z, s, a)=\Phi_{1}(-2 \pi i z, s, a) e^{-2 \pi i a z} .
$$


As is explained in [12], it was A. Erdelyi et al. who first gave the Taylor expansion for $\Phi_{1}(z, s, a)-\Gamma(1-s)(-2 \pi i z)^{s-1}$ with Hurwitz zeta-function coefficients. They applied the functional equation twice, namely, they first used one for $\Phi_{1}(z, s, a)$ and then the Hurwitz formula (the functional equation for the Hurwitz zeta-function). This gives an answer to the evaluation problem in the zeta regularization [4, p.63,(1.31)]. Johnson's argument of applying the residue theorem is more direct and gives the Taylor expansion itself for $\Phi_{A}(-2 \pi i z, s, a)-\frac{1}{A} \Gamma\left(\frac{1-s}{A}\right)(-2 \pi i z)^{\frac{s-1}{A}}$, which gives a closed form for \{\}$_{e x}$ in $[4,(1.28)$, p.63], where \{\}$_{\text {ex }}$ signifies the contribution from the curved part. He does use the functional equation for the Hurwitz zeta-function to show that \{\}$_{e x}=0$. A similar problem with $a=1$ for the divisor function $d(n)=\sum_{d \mid n} 1$ was stated by Hardy [7]:

$$
\sum_{n=1}^{\infty} \frac{d(n)}{n^{s}} e^{-z n^{A}}
$$

which was pursued by Walfisz [19], [20] and then by Kanemitsu [9], and will be elucidated in Concluding Remarks.

Notation. Let $\Gamma(s)$ denote the gamma function. We denote the product $\prod_{j=1}^{K} \Gamma\left(k_{j}\right)$ of $K$ gamma factors by $\Gamma\left(k_{1}, \cdots, k_{K}\right)$, which we further abbreviate by $\Gamma\left(\left\{k_{j}\right\}_{j=1}^{K}\right)$.

We choose the path $L$ in such a way that the poles of the gamma factor

$$
\frac{\Gamma\left(a_{1}-A_{1} s, \ldots, a_{n}-A_{n} s\right)}{\Gamma\left(a_{n+1}-A_{n+1} s, \ldots, a_{p}-A_{p} s\right) \Gamma\left(b_{m+1}-B_{m+1} s, \ldots, b_{q}-B_{q} s\right)}
$$

lie to the right of $L$ and the poles of the gamma factor

$$
\frac{\Gamma\left(b_{1}+B_{1} s, \ldots, b_{m}+B_{m} s\right)}{\Gamma\left(a_{n+1}-A_{n+1} s, \ldots, a_{p}-A_{p} s\right) \Gamma\left(b_{m+1}-B_{m+1} s, \ldots, b_{q}-B_{q} s\right)}
$$

lie to the left of $L$. Then the (Fox) $H$-function is defined by $(0 \leq n \leq p, 0 \leq m \leq q$, $\left.A_{j}, B_{j}>0\right)$

$$
\begin{aligned}
& H_{p, q}^{m, n}\left(z \mid \begin{array}{c}
\left(1-a_{1}, A_{1}\right), \ldots,\left(1-a_{n}, A_{n}\right),\left(a_{n+1}, A_{n+1}\right), \ldots,\left(a_{p}, A_{p}\right) \\
\left(b_{1}, B_{1}\right), \ldots,\left(b_{m}, B_{m}\right),\left(1-b_{m+1}, B_{m+1}\right), \ldots,\left(1-b_{q}, B_{q}\right)
\end{array}\right) \\
& =\frac{1}{2 \pi i} \int_{L} \frac{\Gamma\left(b_{1}+B_{1} s, \ldots, b_{m}+B_{m} s\right) \Gamma\left(a_{1}-A_{1} s, \ldots, a_{n}-A_{n} s\right)}{\Gamma\left(a_{n+1}+A_{n+1} s, \ldots, a_{p}+A_{p} s\right) \Gamma\left(b_{m+1}-B_{m+1} s, \ldots, b_{q}-B_{q} s\right)} z^{-s} d s .
\end{aligned}
$$

On the other hand, the (Meijer) $G$-function is defined as

$$
\begin{aligned}
& G_{p, q}^{m, n}\left(z \mid \begin{array}{c}
1-a_{1}, \ldots, 1-a_{n}, a_{n+1}, \ldots, a_{p} \\
b_{1}, \ldots, b_{m}, 1-b_{m+1}, \ldots, 1-b_{q}
\end{array}\right) \\
& =H_{p, q}^{m, n}\left(z \mid \begin{array}{c}
\left(1-a_{1}, 1\right), \ldots,\left(1-a_{n}, 1\right),\left(a_{n+1}, 1\right), \ldots,\left(a_{p}, 1\right) \\
\left(b_{1}, 1\right), \ldots,\left(b_{m}, 1\right),\left(1-b_{m+1}, 1\right), \ldots,\left(1-b_{q}, 1\right)
\end{array}\right) \\
& =\frac{1}{2 \pi i} \int_{L} \frac{\Gamma\left(b_{1}+s, \ldots, b_{m}+s\right) \Gamma\left(a_{1}-s, \ldots, a_{n}-s\right)}{\Gamma\left(a_{n+1}+s, \ldots, a_{p}+s\right) \Gamma\left(b_{m+1}-s, \ldots, b_{q}-s\right)} z^{-s} d s .
\end{aligned}
$$

(For the properties of $G$ - and $H$-functions, we refer to [5], [6], [16] and [17].) 


\section{The Main Formula}

In order to state Main Formula we first introduce some notation. With increasing sequences $\left\{\lambda_{k}\right\},\left\{\mu_{k}^{(i)}\right\}$ tending to $\infty \quad(1 \leq i \leq I)$ and complex sequences $\left\{\alpha_{k}\right\},\left\{\beta_{k}^{(i)}\right\} \quad(1 \leq$ $i \leq I)$, we form the Dirichlet series

$$
\begin{aligned}
\varphi(s) & =\sum_{k=1}^{\infty} \frac{\alpha_{k}}{\lambda_{k}^{s}}, \\
\psi_{i}(s) & =\sum_{k=1}^{\infty} \frac{\beta_{k}^{(i)}}{\mu_{k}^{(i) s}}, \quad(1 \leq i \leq I)
\end{aligned}
$$

which we suppose have finite abscissas of absolute convergence $\sigma_{\varphi}, \sigma_{\psi_{i}},(1 \leq i \leq I)$, respectively.

We assume the existence of the meromorphic function $\chi$ satisfying the functional equation for a real number $r$ :

$$
\chi(s)=\left\{\begin{array}{l}
\frac{\Gamma\left(\left\{d_{j}+D_{j} s\right\}_{j=1}^{M}\right)}{\Gamma\left(\left\{c_{j}+C_{j} s\right\}_{j=1}^{P}\right)} \varphi(s), \quad \text { if } \Re(s)>\sigma_{\varphi}, \\
\sum_{i=1}^{I} \frac{\Gamma\left(\left\{e_{j}^{(i)}+E_{j}^{(i)}(r-s)\right\}_{j=1}^{\tilde{N}^{(i)}}\right) \Gamma\left(\left\{f_{j}^{(i)}-F_{j}^{(i)}(r-s)\right\}_{j=1}^{\tilde{M}^{(i)}}\right)}{\Gamma\left(\left\{f_{j}^{(i)}+F_{j}^{(i)}(r-s)\right\}_{j=\tilde{Q}^{(i)}+1}^{(i)}\right) \Gamma\left(\left\{e_{j}^{(i)}-E_{j}^{(i)}(r-s)\right\}_{j=\tilde{N}^{(i)}+1}^{\tilde{P}^{(i)}}\right)} \psi_{i}(r-s), \\
\quad \text { if } \Re(s)<\min _{1 \leq i \leq I}\left(r-\sigma_{\psi_{i}}\right),
\end{array}\right.
$$

with $C_{j}, D_{j}, E_{j}^{(i)}, F_{j}^{(i)}>0$.

We assume further that among the poles of the function

$$
\frac{\Gamma\left(\left\{b_{j}-B_{j} s+B_{j} w\right\}_{j=1}^{m}\right)}{\Gamma\left(\left\{a_{j}-A_{j} s+A_{j} w\right\}_{j=n+1}^{p}\right) \Gamma\left(\left\{b_{j}+B_{j} s-B_{j} w\right\}_{j=m+1}^{q}\right)} \chi(w)
$$

in $w$, only finitely many $s_{k}(1 \leq k \leq L)$ are not the poles of

$$
\begin{aligned}
& \frac{\Gamma\left(\left\{b_{j}-B_{j} s+B_{j} w\right\}_{j=1}^{m}\right) \Gamma\left(\left\{f_{j}^{(i)}-F_{j}^{(i)} r+F_{j}^{(i)} w\right\}_{j=1}^{\tilde{M}^{(i)}}\right)}{\Gamma\left(\left\{a_{j}-A_{j} s+A_{j} w\right\}_{j=n+1}^{p}\right) \Gamma\left(\left\{e_{j}^{(i)}-E_{j}^{(i)} r+E_{j}^{(i)} w\right\}_{j=\tilde{N}^{(i)}+1}^{\tilde{P}^{(i)}}\right)} \\
& \times \frac{1}{\Gamma\left(\left\{b_{j}+B_{j} s-B_{j} w\right\}_{j=m+1}^{q}\right) \Gamma\left(\left\{f_{j}^{(i)}+F_{j}^{(i)} r-F_{j}^{(i)} w\right\}_{j=\tilde{M}^{(i)}+1}^{\tilde{Q}^{(i)}}\right)} .
\end{aligned}
$$

We define the gamma quotient

$$
\Delta(w)=\frac{\Gamma\left(\left\{b_{j}+B_{j} w\right\}_{j=1}^{m}\right) \Gamma\left(\left\{a_{j}-A_{j} w\right\}_{j=1}^{n}\right)}{\Gamma\left(\left\{a_{j}+A_{j} w\right\}_{j=n+1}^{p}\right) \Gamma\left(\left\{b_{j}-B_{j} w\right\}_{j=m+1}^{q}\right)} \quad\left(A_{j}, B_{j}>0\right),
$$

and assume the growth condition: for any real numbers $u_{1}, u_{2} \quad\left(u_{1}<u_{2}\right)$, we have

$$
\lim _{|v| \rightarrow \infty} \Delta(u+i v-s) \chi(u+i v)=0
$$


uniformly in $u_{1} \leq u \leq u_{2}$.

We take two Bromwich paths $L_{1}=L_{1}(s), L_{2}=L_{2}(s)$ which are deformed in finite part such a way that the poles of

$$
\frac{\Gamma\left(\left\{a_{j}+A_{j} s-A_{j} w\right\}_{j=1}^{n}\right)}{\Gamma\left(\left\{a_{j}-A_{j} s+A_{j} w\right\}_{j=n+1}^{p}\right) \Gamma\left(\left\{b_{j}+B_{j} s-B_{j} w\right\}_{j=m+1}^{q}\right)}
$$

lie to the right of $L_{1}$, and $s_{k}(1 \leq k \leq L)$ lie to the left of $L_{1}$ and the poles of

$$
\begin{aligned}
& \frac{\Gamma\left(\left\{b_{j}-B_{j} s+B_{j} w\right\}_{j=1}^{m}\right) \Gamma\left(\left\{f_{j}^{(i)}-F_{j}^{(i)} r+F_{j}^{(i)} w\right\}_{j=1}^{\tilde{M}^{(i)}}\right)}{\Gamma\left(\left\{a_{j}-A_{j} s+A_{j} w\right\}_{j=n+1}^{p}\right) \Gamma\left(\left\{e_{j}^{(i)}-E_{j}^{(i)} r+E_{j}^{(i)} w\right\}_{j=\tilde{N}^{(i)}+1}^{\tilde{P}^{(i)}}\right)} \\
& \times \frac{1}{\Gamma\left(\left\{b_{j}+B_{j} s-B_{j} w\right\}_{j=m+1}^{q}\right) \Gamma\left(\left\{f_{j}^{(i)}+F_{j}^{(i)} r-F_{j}^{(i)} w\right\}_{j=\tilde{M}^{(i)}+1}^{\tilde{Q}^{(i)}}\right)}
\end{aligned}
$$

lie to the left of $L_{2}$, those of

$$
\begin{aligned}
& \frac{1}{\Gamma\left(\left\{a_{j}-A_{j} s+A_{j} w\right\}_{j=n+1}^{p}\right) \Gamma\left(\left\{e_{j}^{(i)}-E_{j}^{(i)} r+E_{j}^{(i)} w\right\}_{j=\tilde{N}^{(i)}+1}^{\tilde{P}^{(i)}}\right)} \\
& \times \frac{\Gamma\left(\left\{e_{j}^{(i)}+E_{j}^{(i)} r-E_{j}^{(i)} w\right\}_{j=1}^{(i)}\right)}{\Gamma\left(\left\{b_{j}+B_{j} s-B_{j} w\right\}_{j=m+1}^{q}\right) \Gamma\left(\left\{f_{j}^{(i)}+F_{j}^{(i)} r-F_{j}^{(i)} w\right\}_{j=\tilde{M}^{(i)}+1}^{(i)}\right)}
\end{aligned}
$$

and $s_{k}(1 \leq k \leq L)$ lie to the right of $L_{2}$.

Under these conditions we define the function $\mathrm{X}(z, s)$ by

$$
\mathrm{X}(z, s)=\frac{1}{2 \pi i} \int_{L_{1}} \Delta(w-s) \chi(w) z^{-w} d w
$$

Then, we have

$$
\mathrm{X}(z, s)=\frac{1}{2 \pi i} \int_{L_{2}} \Delta(w-s) \chi(w) z^{-w} d w+\frac{1}{2 \pi i} \int_{\mathcal{C}} \Delta(w-s) \chi(w) z^{-w} d w,
$$

where $\mathcal{C}$ is the boundary of a compact set containing $s_{k}(1 \leq k \leq L)$, and we have a form of the functional equation (a variation of the general functional equation in [18]): 


\section{The Ramified Type Formula $\mathbf{H}$ :}

$\mathrm{X}(z, s)$

$$
=\left\{\begin{array}{c}
\sum_{k=1}^{\infty} \alpha_{k} H_{p+P, q+M}^{m+M, n}\left(z \lambda_{k} \mid \begin{array}{c}
\left\{\left(1-a_{j}-A_{j} s, A_{j}\right)\right\}_{j=1}^{n},\left\{\left(a_{j}-A_{j} s, A_{j}\right)\right\}_{j=n+1}^{p},\left\{\left(c_{j}, C_{j}\right)\right\}_{j=1}^{P} \\
\left\{\left(b_{j}-B_{j} s, B_{j}\right)\right\}_{j=1}^{m},\left\{\left(d_{j}, D_{j}\right)\right\}_{j=1}^{M},\left\{\left(1-b_{j}-B_{j} s, B_{j}\right)\right\}_{j=m+1}^{q}
\end{array}\right. \\
\text { if } L_{1} \text { can be taken to the right of } \sigma_{\varphi},
\end{array}\right)
$$

or equivalently,

$z^{s} \mathrm{X}(z, s)$

$$
\begin{aligned}
& \left(\begin{array}{l|l}
\sum_{k=1}^{\infty} \frac{\alpha_{k}}{\lambda_{k}^{s}} H_{p+P, q+M}^{m+M, n} \\
z \lambda_{k} & \begin{array}{l}
\left\{\left(1-a_{j}, A_{j}\right)\right\}_{j=1}^{n},\left\{\left(a_{j}, A_{j}\right)\right\}_{j=n+1}^{p},\left\{\left(c_{j}+C_{j} s, C_{j}\right)\right\}_{j=1}^{P} \\
\left\{\left(b_{j}, B_{j}\right)\right\}_{j=1}^{m},\left\{\left(d_{j}+D_{j} s, D_{j}\right)\right\}_{j=1}^{M},\left\{\left(1-b_{j}, B_{j}\right)\right\}_{j=m+1}^{q}
\end{array}
\end{array}\right)
\end{aligned}
$$

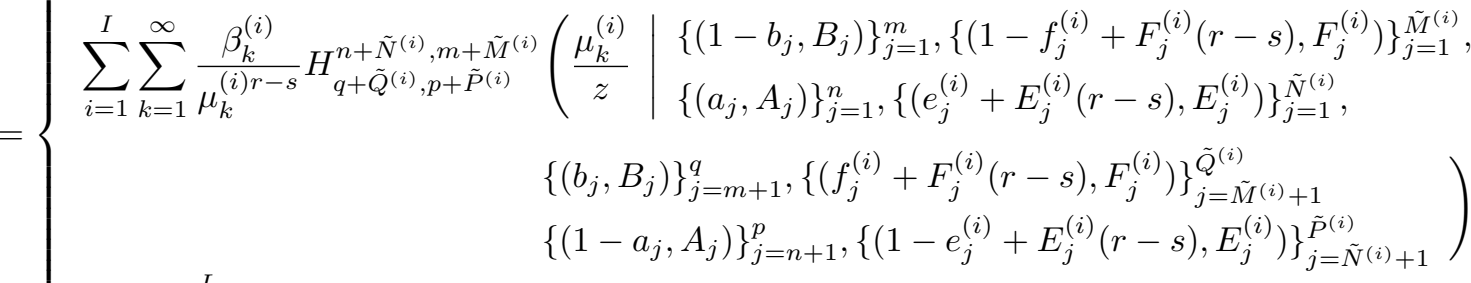

$$
\begin{aligned}
& +\sum_{k=1}^{L} \operatorname{Res}\left(\Delta(w-s) \chi(w) z^{s-w}, w=s_{k}\right)
\end{aligned}
$$

$$
\left(\begin{array}{c}
\sum_{j=1}^{n} A_{j}+\sum_{j=1}^{m} B_{j}+\sum_{j=1}^{M} D_{j} \geq \sum_{j=n+1}^{p} A_{j}+\sum_{j=m+1}^{q} B_{j}+\sum_{j=1}^{P} C_{j}, \\
\sum_{j=1}^{n} A_{j}+\sum_{j=1}^{m} B_{j}+\sum_{j=1}^{\tilde{N}^{(i)}} E_{j}^{(i)}+\sum_{j=1}^{\tilde{M}^{(i)}} F_{j}^{(i)} \\
\quad \geq \sum_{j=n+1}^{p} A_{j}+\sum_{j=m+1}^{q} B_{j}+\sum_{j=\tilde{N}^{(i)}+1}^{q(i)} E_{j}^{(i)}+\sum_{j=\tilde{M}^{(i)}+1}^{\tilde{Q}^{(i)}} F_{j}^{(i)}
\end{array}\right) .
$$

In the special case $A_{j}=B_{j}=C_{j}=D_{j}=E_{j}^{(i)}=F_{j}^{(i)}=1$, we have

$$
\Delta(w)=\frac{\Gamma\left(\left\{b_{j}+w\right\}_{j=1}^{m}\right) \Gamma\left(\left\{a_{j}-w\right\}_{j=1}^{n}\right)}{\Gamma\left(\left\{a_{j}+w\right\}_{j=n+1}^{p}\right) \Gamma\left(\left\{b_{j}-w\right\}_{j=m+1}^{q}\right)},
$$


and the Ramified Type Formula $\mathrm{H}$ reduces to

\section{The Ramified Type Formula G:}

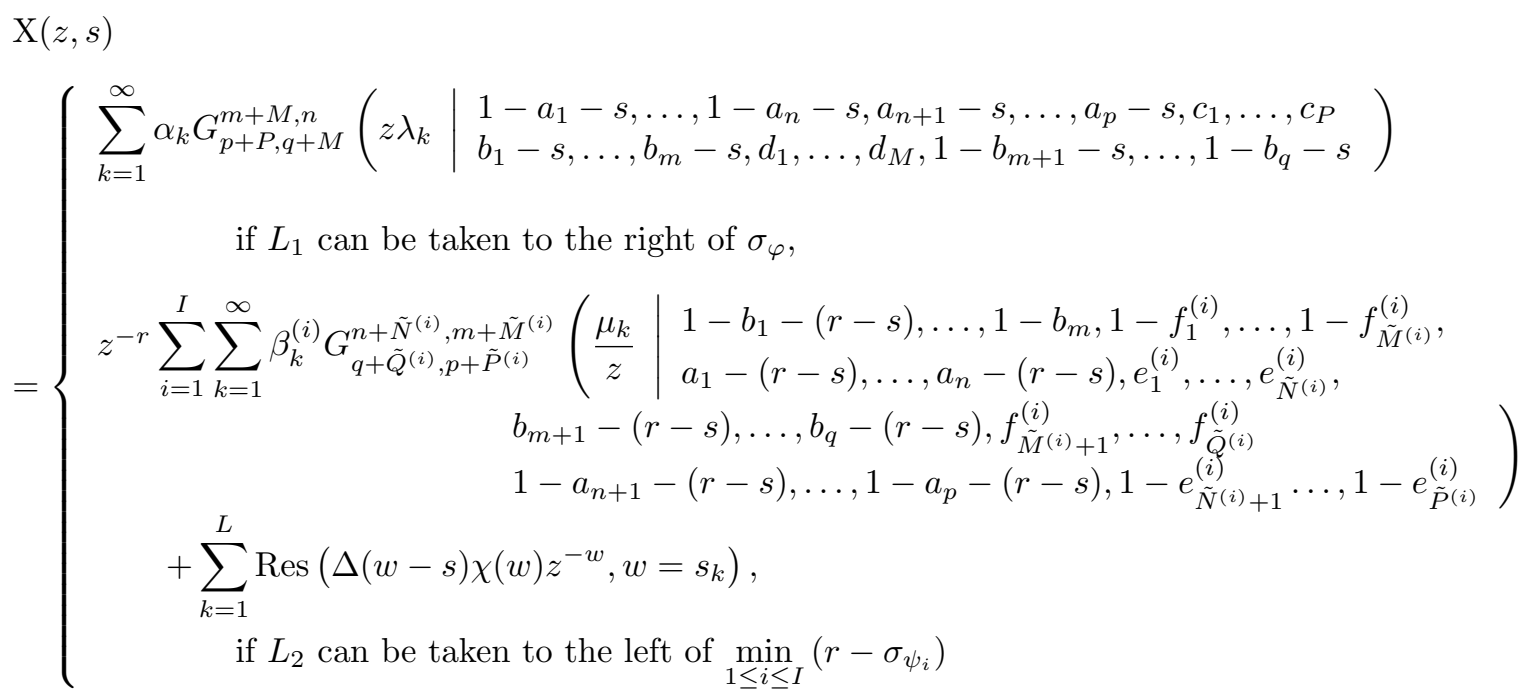

or equivalently,

$$
\begin{aligned}
& z^{s} \mathrm{X}(z, s)
\end{aligned}
$$

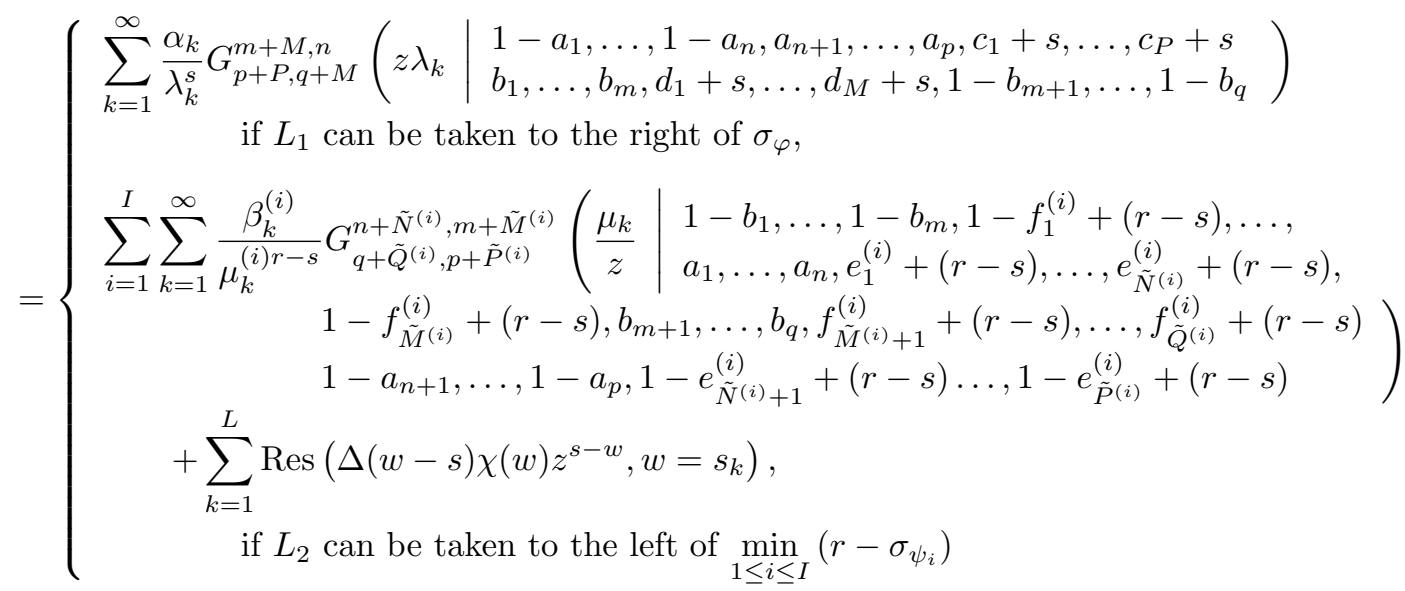

$$
\begin{aligned}
& (2 n+2 m+M \geq p+q+P, 2 n+2 m+2 \tilde{N}+2 \tilde{M} \geq p+q+\tilde{P}+\tilde{Q}) .
\end{aligned}
$$

Proof of the Ramified Type Formula $H$. We note that all the poles of

$$
\frac{\Gamma\left(\left\{a_{j}+A_{j} s-A_{j} w\right\}_{j=1}^{n}\right)}{\Gamma\left(\left\{a_{j}-A_{j} s+A_{j} w\right\}_{j=n+1}^{p},\left\{c_{j}+C_{j} w\right\}_{j=1}^{P}\right) \Gamma\left(\left\{b_{j}+B_{j} s-B_{j} w\right\}_{j=m+1}^{q}\right)}
$$

lie to the right of $L_{1}$ and those of

$$
\frac{\Gamma\left(\left\{b_{j}-B_{j} s+B_{j} w\right\}_{j=1}^{m},\left\{d_{j}+D_{j} w\right\}_{j=1}^{M}\right)}{\Gamma\left(\left\{a_{j}-A_{j} s+A_{j} w\right\}_{j=n+1}^{p},\left\{c_{j}+C_{j} w\right\}_{j=1}^{P}\right) \Gamma\left(\left\{b_{j}+B_{j} s-B_{j} w\right\}_{j=m+1}^{q}\right)}
$$


lie to the left of $L_{1}$. Hence, if the condition on $L_{1}$ is satisfied, then we obtain

$$
\begin{aligned}
& \mathrm{X}(z, s) \\
& \begin{array}{r}
=\frac{1}{2 \pi i} \int_{L_{1}} \frac{\Gamma\left(\left\{b_{j}-B_{j} s+B_{j} w\right\}_{j=1}^{m}\right) \Gamma\left(\left\{a_{j}+A_{j} s-A_{j} w\right\}_{j=1}^{n}\right)}{\Gamma\left(\left\{a_{j}-A_{j} s+A_{j} w\right\}_{j=n+1}^{p}\right) \Gamma\left(\left\{b_{j}+B_{j} s-B_{j} w\right\}_{j=m+1}^{q}\right)} \\
\times \frac{\Gamma\left(\left\{d_{j}+D_{j} w\right\}_{j=1}^{M}\right)}{\Gamma\left(\left\{c_{j}+C_{j} w\right\}_{j=1}^{P}\right)} \varphi(w) z^{-w} d w \\
=\frac{1}{2 \pi i} \int_{L_{1}} \frac{\Gamma\left(\left\{b_{j}-B_{j} s+B_{j} w\right\}_{j=1}^{m},\left\{d_{j}+D_{j} w\right\}_{j=1}^{M}\right)}{\Gamma\left(\left\{a_{j}-A_{j} s+A_{j} w\right\}_{j=n+1}^{p},\left\{c_{j}+C_{j} w\right\}_{j=1}^{P}\right)} \\
\times \frac{\Gamma\left(\left\{a_{j}+A_{j} s-A_{j} w\right\}_{j=1}^{n}\right)}{\Gamma\left(\left\{b_{j}+B_{j} s-B_{j} w\right\}_{j=m+1}^{q}\right)} \sum_{k=1}^{\infty} \frac{\alpha_{k}}{\left(z \lambda_{k}\right)^{w}} d w \\
=\sum_{k=1}^{\infty} \alpha_{k} H_{p+P, q+M}^{m+M, n}\left(z \lambda_{k} \mid \begin{array}{l}
\left\{\left(1-a_{j}-A_{j} s, A_{j}\right)\right\}_{j=1}^{n},\left\{\left(a_{j}-A_{j} s, A_{j}\right)\right\}_{j=n+1}^{p},\left\{\left(c_{j}, C_{j}\right)\right\}_{j=1}^{P} \\
\left\{\left(b_{j}-B_{j} s, B_{j}\right)\right\}_{j=1}^{m},\left\{\left(d_{j}, D_{j}\right)\right\}_{j=1}^{M},\left\{\left(1-b_{j}-B_{j} s, B_{j}\right)\right\}_{j=m+1}^{q}
\end{array}\right) .
\end{array}
\end{aligned}
$$

On the other hand, all the poles of

$$
\begin{aligned}
& \frac{1}{\Gamma\left(\left\{a_{j}-A_{j} s+A_{j} w\right\}_{j=n+1}^{p},\left\{e_{j}^{(i)}-E_{j}^{(i)} r+E_{j}^{(i)} w\right\}_{j=\tilde{N}^{(i)}+1}^{\tilde{P}^{(i)}}\right)} \\
& \times \frac{\Gamma\left(\left\{a_{j}+A_{j} s-A_{j} w\right\}_{j=1}^{n},\left\{e_{j}^{(i)}+E_{j}^{(i)} r-E_{j}^{(i)} w\right\}_{j=1}^{(i)}\right)}{\Gamma\left(\left\{b_{j}+B_{j} s-B_{j} w\right\}_{j=m+1}^{q},\left\{f_{j}^{(i)}+F_{j}^{(i)} r-F_{j}^{(i)} w\right\}_{j=\tilde{M}^{(i)}+1}^{(i)}\right)}
\end{aligned}
$$

lie to the right of $L_{2}$, and those of

$$
\begin{aligned}
& \frac{\Gamma\left(\left\{b_{j}-B_{j} s+B_{j} w\right\}_{j=1}^{m},\left\{f_{j}^{(i)}-F_{j}^{(i)} r+F_{j}^{(i)} w\right\}_{j=1}^{\tilde{M}^{(i)}}\right)}{\Gamma\left(\left\{a_{j}-A_{j} s+A_{j} w\right\}_{j=n+1}^{p},\left\{e_{j}^{(i)}-E_{j}^{(i)} r+E_{j}^{(i)} w\right\}_{j=\tilde{N}^{(i)}+1}^{(i)}\right)} \\
& \times \frac{1}{\Gamma\left(\left\{b_{j}+B_{j} s-B_{j} w\right\}_{j=m+1}^{q},\left\{f_{j}^{(i)}+F_{j}^{(i)} r-F_{j}^{(i)} w\right\}_{j=\tilde{M}^{(i)}+1}^{(i)}\right)}
\end{aligned}
$$

lie to the left of $L_{2}$. Hence under the condition on $L_{2}$, we have

$$
\begin{aligned}
& \mathrm{X}(z, s) \\
& =\frac{1}{2 \pi i} \int_{L_{2}} \frac{\Gamma\left(\left\{b_{j}-B_{j} s+B_{j} w\right\}_{j=1}^{m}\right) \Gamma\left(\left\{a_{j}+A_{j} s-A_{j} w\right\}_{j=1}^{n}\right)}{\Gamma\left(\left\{a_{j}-A_{j} s+A_{j} w\right\}_{j=n+1}^{p}\right) \Gamma\left(\left\{b_{j}+B_{j} s-B_{j} w\right\}_{j=m+1}^{q}\right)} \\
& \quad \times \sum_{i=1}^{I} \frac{\Gamma\left(\left\{f_{j}^{(i)}-F_{j}^{(i)} r+F_{j}^{(i)} w\right\}_{j=1}^{\tilde{M}^{(i)}}\right) \Gamma\left(\left\{e_{j}^{(i)}+E_{j}^{(i)} r-E_{j}^{(i)} w\right\}_{j=1}^{\tilde{N}^{(i)}}\right)}{\Gamma\left(\left\{e_{j}^{(i)}-E_{j}^{(i)} r+E_{j}^{(i)} w\right\}_{j=\tilde{N}^{(i)}+1}^{\tilde{P}^{(i)}}\right) \Gamma\left(\left\{f_{j}^{(i)}+F_{j}^{(i)} r-F_{j}^{(i)} w\right\}_{j=\tilde{M}^{(i)}+1}^{\tilde{Q}^{(i)}}\right)} \psi_{i}(r-w) z^{-w} d w
\end{aligned}
$$




$$
\begin{aligned}
& +\sum_{k=1}^{L} \operatorname{Res}\left(\Delta(w-s) \chi(s) z^{-w}, w=s_{k}\right) \\
& =z^{-r} \frac{1}{2 \pi i} \sum_{i=1}^{I} \int_{L_{2}} \frac{\Gamma\left(\left\{b_{j}-B_{j} s+B_{j} w\right\}_{j=1}^{m},\left\{f_{j}^{(i)}-F_{j}^{(i)} r+F_{j}^{(i)} w\right\}_{j=1}^{\tilde{M}^{(i)}}\right)}{\Gamma\left(\left\{a_{j}-A_{j} s+A_{j} w\right\}_{j=n+1}^{p},\left\{e_{j}^{(i)}-E_{j}^{(i)} r+E_{j}^{(i)} w\right\}_{j=\tilde{N}^{(i)}+1}^{\tilde{P}^{(i)}}\right)} \\
& \times \frac{\Gamma\left(\left\{a_{j}+A_{j} s-A_{j} w\right\}_{j=1}^{n},\left\{e_{j}^{(i)}+E_{j}^{(i)} r-E_{j}^{(i)} w\right\}_{j=1}^{\tilde{N}^{(i)}}\right)}{\Gamma\left(\left\{b_{j}+B_{j} s-B_{j} w\right\}_{j=m+1}^{q},\left\{f_{j}^{(i)}+F_{j}^{(i)} r-F_{j}^{(i)} w\right\}_{j=\tilde{M}^{(i)}+1}^{\tilde{Q}^{(i)}}\right)} \sum_{k=1}^{\infty} \frac{\beta_{k}^{(i)}}{\left(\frac{z}{\mu_{k}^{(i)}}\right)^{w-r}} d w \\
& +\sum_{k=1}^{L} \operatorname{Res}\left(\Delta(w-s) \chi(w) z^{-w}, w=s_{k}\right)
\end{aligned}
$$

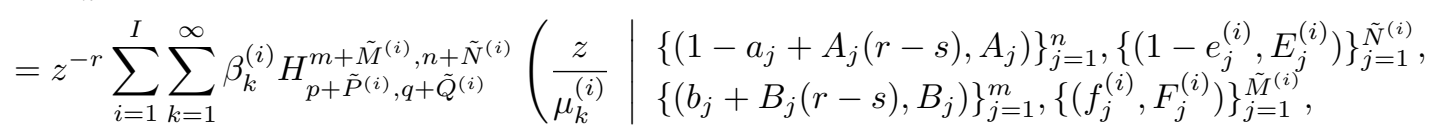

$$
\begin{aligned}
& \left.\begin{array}{l}
\left\{\left(a_{j}+A_{j}(r-s), A_{j}\right)\right\}_{j=n+1}^{p},\left\{\left(e_{j}^{(i)}, E_{j}^{(i)}\right)\right\}_{j=\tilde{N}^{(i)}+1}^{\tilde{P}^{(i)}} \\
\left\{\left(1-b_{j}+B_{j}(r-s), B_{j}\right)\right\}_{j=m+1}^{q},\left\{\left(1-f_{j}^{(i)}, F_{j}^{(i)}\right)\right\}_{j=\tilde{M}^{(i)}+1}^{\tilde{Q}^{(i)}}
\end{array}\right) \\
& +\sum_{k=1}^{L} \operatorname{Res}\left(\Delta(w-s) \chi(w) z^{-w}, w=s_{k}\right) \\
& =z^{-r} \sum_{i=1}^{I} \sum_{k=1}^{\infty} \beta_{k}^{(i)} H_{q+\tilde{Q}^{(i)}, p+\tilde{P}^{(i)}}^{n+\tilde{N}^{(i)}}\left(\frac{\tilde{\mu}_{k}^{(i)}}{z} \mid \begin{array}{l}
\left\{\left(1-b_{j}-B_{j}(r-s), B_{j}\right)\right\}_{j=1}^{m},\left\{\left(1-f_{j}^{(i)}, F_{j}^{(i)}\right)\right\}_{j=1}^{\tilde{M}^{(i)}} \\
\left\{\left(a_{j}-A_{j}(r-s), A_{j}\right)\right\}_{j=1}^{n},\left\{\left(e_{j}^{(i)}, E_{j}^{(i)}\right)\right\}_{j=1}^{\tilde{N}^{(i)}},
\end{array}\right. \\
& \left.\begin{array}{l}
\left\{\left(b_{j}-B_{j}(r-s), B_{j}\right)\right\}_{j=m+1}^{q},\left\{\left(f_{j}^{(i)}, F_{j}^{(i)}\right)\right\}_{j=\tilde{M}^{(i)}+1}^{\tilde{Q}^{(i)}} \\
\left\{\left(1-a_{j}-A_{j}(r-s), A_{j}\right)\right\}_{j=n+1}^{p},\left\{\left(1-e_{j}^{(i)}, E_{j}^{(i)}\right)\right\}_{j=\tilde{N}^{(i)}+1}^{\tilde{P}^{(i)}}
\end{array}\right) \\
& +\sum_{k=1}^{L} \operatorname{Res}\left(\Delta(w-s) \chi(w) z^{-w}, w=s_{k}\right),
\end{aligned}
$$

whence (2.7) follows.

Example. Let

$$
l_{s}(z)=\sum_{k=1}^{\infty} \frac{e^{2 \pi i k z}}{k^{s}}
$$

be the Lerch zeta-function. Then for $0<a<1$, we have the $\mathrm{L} \rightarrow \mathrm{H}$ (Lerch $\rightarrow$ Hurwitz) formula

$$
l_{s}(a)=\frac{\Gamma(1-s)}{(2 \pi)^{1-s}}\left(e^{\frac{1-s}{2} \pi i} \zeta(1-s, 1-a)+e^{-\frac{1-s}{2} \pi i} \zeta(1-s, a)\right) .
$$

Now we appeal to Euler's formula in the form

$$
e^{s \pi i}=\cos (\pi s)+i \sin (\pi s)=\frac{\pi}{\Gamma\left(s+\frac{1}{2}\right) \Gamma\left(\frac{1}{2}-s\right)}+i \frac{\pi}{\Gamma(s) \Gamma(1-s)}
$$

so as to replace the exponentials in (2.10) by gamma functions, deducing in the first place

$$
\frac{1}{\pi^{\frac{s}{2}}} l_{s}(a)=\frac{\Gamma\left(\frac{1}{2}-\frac{s}{2}\right)}{\Gamma\left(\frac{s}{2}\right)}\left(\frac{1}{2 \pi^{\frac{1-s}{2}}} \zeta(1-s, a)+\frac{1}{2 \pi^{\frac{1-s}{2}}} \zeta(1-s, 1-a)\right)
$$




$$
+\frac{\Gamma\left(1-\frac{s}{2}\right)}{\Gamma\left(\frac{1}{2}+\frac{s}{2}\right)}\left(\frac{i}{2 \pi^{\frac{1-s}{2}}} \zeta(1-s, a)-\frac{i}{2 \pi^{\frac{1-s}{2}}} \zeta(1-s, 1-a)\right),
$$

which we further transform into

$$
\begin{aligned}
\frac{1}{\pi^{\frac{s}{2}}} l_{s}(a)= & \frac{\Gamma\left(\frac{1}{2}-\frac{s}{2}\right)}{\Gamma\left(\frac{s}{2}\right)}\left(\frac{1}{2 \pi^{\frac{1-s}{2}}} \zeta(1-s, a)+\frac{1}{2 \pi^{\frac{1-s}{2}}} \zeta(1-s, 1-a)\right) \\
& +\frac{\Gamma\left(1+\frac{s}{2}\right) \Gamma\left(-\frac{s}{2}\right)}{\Gamma\left(\frac{s}{2}\right) \Gamma\left(\frac{1}{2}+\frac{s}{2}\right)}\left(-\frac{i}{2 \pi^{\frac{1-s}{2}}} \zeta(1-s, a)+\frac{i}{2 \pi^{\frac{1-s}{2}}} \zeta(1-s, 1-a)\right) .
\end{aligned}
$$

We interpret (2.12) as in (2.3), for which we use the data:

For $\lambda_{k}=\sqrt{\pi} k, \quad \alpha_{k}=e^{2 \pi i k a}$, we have $\varphi(s)=\frac{1}{\pi^{\frac{s}{2}}} l_{s}(w) \quad$ and $\sigma_{\varphi}=1$, for $\mu_{k}^{(1)}=\sqrt{\pi}(k+a-1), \quad \beta_{k}^{(1)}=\frac{1}{2}, \quad$ we have $\quad \psi_{1}(s)=\frac{1}{2 \pi^{\frac{s}{2}}} \zeta(s, a) \quad$ and $\sigma_{\psi_{1}}=1$, for $\mu_{k}^{(2)}=\sqrt{\pi}(k-a), \quad \beta_{k}^{(2)}=\frac{1}{2}, \quad$ we have $\quad \psi_{2}(s)=\frac{1}{2 \pi^{\frac{s}{2}}} \zeta(s, 1-a)$ and $\sigma_{\psi_{2}}=1$, for $\mu_{k}^{(3)}=\sqrt{\pi}(k+a-1), \quad \beta_{k}^{(3)}=-\frac{i}{2}, \quad$ we have $\quad \psi_{3}(s)=-\frac{i}{2 \pi^{\frac{s}{2}}} \zeta(s, a) \quad$ and $\sigma_{\psi_{3}}=1$, for $\mu_{k}^{(4)}=\sqrt{\pi}(k-a), \quad \beta_{k}^{(4)}=\frac{i}{2}, \quad$ we have $\quad \psi_{4}(s)=\frac{i}{2 \pi^{\frac{5}{2}}} \zeta(s, 1-a)$ and $\sigma_{\psi_{4}}=1$.

Thus, (2.12) is a special case of (2.3) with $r=1$ :

$$
\begin{aligned}
\chi(s)=\varphi(s)= & \frac{\Gamma\left(\frac{1}{2}-\frac{s}{2}\right)}{\Gamma\left(\frac{s}{2}\right)} \psi_{1}(1-s)+\frac{\Gamma\left(\frac{1}{2}-\frac{s}{2}\right)}{\Gamma\left(\frac{s}{2}\right)} \psi_{2}(1-s) \\
& +\frac{\Gamma\left(1+\frac{1}{2}\right) \Gamma\left(-\frac{s}{2}\right)}{\Gamma\left(\frac{s}{2}\right) \Gamma\left(\frac{1}{2}+\frac{s}{2}\right)} \psi_{3}(1-s)+\frac{\Gamma\left(1+\frac{1}{2}\right) \Gamma\left(-\frac{s}{2}\right)}{\Gamma\left(\frac{s}{2}\right) \Gamma\left(\frac{1}{2}+\frac{s}{2}\right)} \psi_{4}(1-s) .
\end{aligned}
$$

We have to check the condition on poles. The function $\chi$ has no pole and among the poles of $\Gamma(w) \chi(w)$, those which are neither the poles of $\frac{\Gamma(w)}{\Gamma\left(\frac{w}{2}\right)}$ nor of $\frac{\Gamma(w) \Gamma\left(1+\frac{w}{2}\right)}{\Gamma\left(\frac{w}{2}\right) \Gamma\left(\frac{1}{2}+\frac{w}{2}\right)}$ is only at $s_{1}=0$.

Thus the Ramified Type Formula H applies to give

$$
\begin{aligned}
& \sum_{k=1}^{\infty} \frac{\alpha_{k}}{\lambda_{k}^{s}} H_{1,1}^{1,1}\left(\begin{array}{l|l}
z \lambda_{k} & \begin{array}{c}
(1,1) \\
(s, 1)
\end{array}
\end{array}\right) \\
& =\sum_{k=1}^{\infty} \frac{\beta_{k}^{(1)}}{\mu_{k}^{(1) 1-s}} H_{1,3}^{2,1}\left(\frac{\mu_{k}^{(1)}}{z} \mid \begin{array}{c}
(1-s, 1) \\
\left(\frac{1}{2}-\frac{s}{2}, \frac{1}{2}\right),(0,1),\left(1-\frac{s}{2}, \frac{1}{2}\right)
\end{array}\right) \\
& +\sum_{k=1}^{\infty} \frac{\beta_{k}^{(2)}}{\mu_{k}^{(2) 1-s}} H_{1,3}^{2,1}\left(\frac{\mu_{k}^{(2)}}{z} \mid \begin{array}{c}
(1-s, 1) \\
\left(\frac{1}{2}-\frac{s}{2}, \frac{1}{2}\right),(0,1),\left(1-\frac{s}{2}, \frac{1}{2}\right)
\end{array}\right) \\
& +\sum_{k=1}^{\infty} \frac{\beta_{k}^{(3)}}{\mu_{k}^{(3) 1-s}} H_{2,4}^{2,2}\left(\frac{\mu_{k}^{(3)}}{z} \mid \begin{array}{c}
\left(-\frac{s}{2}, \frac{1}{2}\right),(1-s, 1) \\
\left(-\frac{s}{2}, \frac{1}{2}\right),(0,1),\left(1-\frac{s}{2}, \frac{1}{2}\right),\left(\frac{1}{2}-\frac{s}{2}, \frac{1}{2}\right)
\end{array}\right) \\
& +\sum_{k=1}^{\infty} \frac{\beta_{k}^{(4)}}{\mu_{k}^{(4) 1-s}} H_{2,4}^{2,2}\left(\frac{\mu_{k}^{(4)}}{z} \mid \begin{array}{c}
\left(-\frac{s}{2}, \frac{1}{2}\right),(1-s, 1) \\
\left(-\frac{s}{2}, \frac{1}{2}\right),(0,1),\left(1-\frac{s}{2}, \frac{1}{2}\right),\left(\frac{1}{2}-\frac{s}{2}, \frac{1}{2}\right)
\end{array}\right)
\end{aligned}
$$




$$
+\operatorname{Res}\left(\Gamma(w) \Gamma(s-w) \chi(w) z^{s-w}, w=s_{1}\right)
$$

By $[17,8.3 .2 .21$, p.629], the left-hand side of (2.14) becomes

$$
\sum_{k=1}^{\infty} \frac{\alpha_{k}}{\lambda_{k}^{s}} G_{1,1}^{1,1}\left(z \lambda_{k} \mid \begin{array}{l}
1 \\
s
\end{array}\right)
$$

We appeal to the special cases of the formulas for $H$-functions. First, by $[17,8.4 .2 .5$, p. 631]

$$
G_{1,1}^{1,1}\left(\begin{array}{l|l}
z & 1 \\
s
\end{array}\right)=\Gamma(s) \frac{z^{s}}{(1+z)^{s}} .
$$

By the duplication formula, we have

$$
\begin{aligned}
& H_{1,3}^{2,1}\left(\begin{array}{l|c}
z \mid & (1-s, 1) \\
\left(\frac{1-s}{2}, \frac{1}{2}\right),(0,1),\left(1-\frac{s}{2}, \frac{1}{2}\right)
\end{array}\right) \\
& =\frac{1}{2^{2-s} \pi} H_{1,3}^{3,1}\left(\begin{array}{l|c}
z & \left(\frac{1-s}{2}, \frac{1}{2}\right) \\
\left(\frac{1-s}{2}, \frac{1}{2}\right),\left(0, \frac{1}{2}\right),\left(\frac{1}{2}, \frac{1}{2}\right)
\end{array}\right)=\frac{1}{2^{1-s} \pi} G_{1,3}^{3,1}\left(\begin{array}{l|c}
z^{2} & \frac{1-s}{2} \\
\frac{1-s}{2}, 0, \frac{1}{2}
\end{array}\right)
\end{aligned}
$$

by $[17,8.3 .2 .22$, p. 621$]$, which by $[17,8.4 .16 .18$, p. 649$]$ becomes

$$
=\frac{\Gamma(s)}{2^{1-s} \sqrt{\pi}}\left(e^{\frac{1-s}{2} \pi i-2 z i} \Gamma(1-s,-2 z i)+e^{-\frac{1-s}{2} \pi i+2 z i} \Gamma(1-s, 2 z i)\right),
$$

where and hereafter until the end of this section, $\Gamma(s, z)$ indicates the incomplete gamma function of the second kind defined by

$$
\Gamma(s, z)=\int_{z}^{\infty} e^{-u} u^{s-1} d u, \quad \Re s>0,|\arg z|<\pi
$$

and

$$
\begin{aligned}
& H_{2,4}^{2,2}\left(z \mid \begin{array}{c}
\left(-\frac{s}{2}, \frac{1}{2}\right),(1-s, 1) \\
\left(-\frac{s}{2}, \frac{1}{2}\right),\left(0, \frac{1}{1}\right),\left(1-\frac{s}{2}, \frac{1}{2}\right),\left(\frac{1}{2}-\frac{s}{2}, \frac{1}{2}\right)
\end{array}\right) \\
& =\frac{1}{2^{2-s} \pi} H_{1,3}^{3,1}\left(z \mid \begin{array}{c}
\left(-\frac{s}{2}, \frac{1}{2}\right) \\
\left(-\frac{s}{2}, \frac{1}{2}\right),\left(0, \frac{1}{2}\right),\left(\frac{1}{2}, \frac{1}{2}\right)
\end{array}\right) \\
& =\frac{1}{2^{1-s} \pi} G_{1,3}^{3,1}\left(z^{2} \mid \begin{array}{c}
-\frac{s}{2} \\
-\frac{s}{2}, 0, \frac{1}{2}
\end{array}\right) \\
& =\frac{\Gamma(s+1)}{2^{1-s} \sqrt{\pi}}\left(e^{-\frac{s}{2} \pi i-2 z i} \Gamma(-s,-2 z i)+e^{\frac{s}{2} \pi i+2 z i} \Gamma(-s, 2 z i)\right) \\
& =\frac{\Gamma(s)}{2^{1-s} \sqrt{\pi}}\left(i e^{\frac{1-s}{2} \pi i-2 z i} \Gamma(1-s,-2 z i)-i e^{-\frac{1-s}{2} \pi i+2 z i} \Gamma(1-s, 2 z i)\right)+\frac{\Gamma(s)}{z^{s} \sqrt{\pi}}
\end{aligned}
$$

Hence, formula (2.14) with $z$ replaced by $\frac{1}{z}$ reads

$$
\Gamma(s) \sum_{k=1}^{\infty} \frac{\alpha_{k}}{\left(\lambda_{k}+z\right)^{s}}
$$




$$
\begin{aligned}
& =\frac{\Gamma(s)}{z^{s-1} \sqrt{\pi}} \sum_{k=1}^{\infty} \beta_{k}^{(1)}\left(\frac{e^{-2 \mu_{k}^{(1)} z i}}{\left(2 \mu_{k}^{(1)} z e^{-\frac{\pi i}{2}}\right)^{1-s}} \Gamma\left(1-s,-2 \mu_{k}^{(1)} z i\right)\right. \\
& \left.+\frac{e^{2 \mu_{k}^{(1)} z i}}{\left(2 \mu_{k}^{(1)} z e^{\frac{\pi i}{2}}\right)^{1-s}} \Gamma\left(1-s, 2 \mu_{k}^{(1)} z i\right)\right) \\
& +\frac{\Gamma(s)}{z^{s-1} \sqrt{\pi}} \sum_{k=1}^{\infty} \beta_{k}^{(2)}\left(\frac{e^{-2 \mu_{k}^{(2)} z i}}{\left(2 \mu_{k}^{(2)} z e^{-\frac{\pi i}{2}}\right)^{1-s}} \Gamma\left(1-s,-2 \mu_{k}^{(2)} z i\right)\right. \\
& \left.+\frac{e^{2 \mu_{k}^{(2)} z i}}{\left(2 \mu_{k}^{(2)} z e^{\frac{\pi i}{2}}\right)^{1-s}} \Gamma\left(1-s, 2 \mu_{k}^{(2)} z i\right)\right) \\
& +\frac{\Gamma(s)}{z^{s-1} \sqrt{\pi}} \sum_{k=1}^{\infty} \beta_{k}^{(3)}\left(\frac{i e^{-2 \mu_{k}^{(3)} z i}}{\left(2 \mu_{k}^{(3)} z e^{-\frac{\pi i}{2}}\right)^{1-s}} \Gamma\left(1-s,-2 \mu_{k}^{(3)} z i\right)\right. \\
& \left.-\frac{i e^{2 \mu_{k}^{(3)} z i}}{\left(2 \mu_{k}^{(3)} z e^{\frac{\pi i}{2}}\right)^{1-s}} \Gamma\left(1-s, 2 \mu_{k}^{(3)} z i\right)+\frac{1}{z \mu_{k}^{(3)}}\right) \\
& +\frac{\Gamma(s)}{z^{s-1} \sqrt{\pi}} \sum_{k=1}^{\infty} \beta_{k}^{(4)}\left(\frac{i e^{-2 \mu_{k}^{(4)} z i}}{\left(2 \mu_{k}^{(4)} z e^{-\frac{\pi i}{2}}\right)^{1-s}} \Gamma\left(1-s,-2 \mu_{k}^{(4)} z i\right)\right. \\
& \left.-\frac{i e^{2 \mu_{k}^{(4)} z i}}{\left(2 \mu_{k}^{(4)} z e^{\frac{\pi i}{2}}\right)^{1-s}} \Gamma\left(1-s, 2 \mu_{k}^{(4)} z i\right)+\frac{1}{z \mu_{k}^{(4)}}\right) \\
& +\operatorname{Res}\left(\Gamma(w) \Gamma(s-w) \chi(w) z^{w-s}, w=s_{1}\right) \text {. }
\end{aligned}
$$

Or, more concretely,

$$
\begin{aligned}
& \sum_{k=1}^{\infty} \frac{e^{2 \pi i k a}}{(\sqrt{\pi} k+z)^{s}} \\
& =\frac{1}{2 z^{s-1} \sqrt{\pi}} \sum_{k=1}^{\infty}\left(\frac{e^{-2 \sqrt{\pi}(k+a-1) z i}}{\left(2 \sqrt{\pi}(k+a-1) z e^{-\frac{\pi i}{2}}\right)^{1-s}} \Gamma(1-s,-2 \sqrt{\pi}(k+a-1) z i)\right. \\
& \left.+\frac{e^{2 \sqrt{\pi}(k+a-1) z i}}{\left(2 \sqrt{\pi}(k+a-1) z e^{\frac{\pi i}{2}}\right)^{1-s}} \Gamma(1-s, 2 \sqrt{\pi}(k+a-1) z i)\right) \\
& +\frac{1}{2 z^{s-1} \sqrt{\pi}} \sum_{k=1}^{\infty}\left(\frac{e^{-2 \sqrt{\pi}(k-a) z i}}{\left(2 \sqrt{\pi}(k-a) z e^{-\frac{\pi i}{2}}\right)^{1-s}} \Gamma(1-s,-2 \sqrt{\pi}(k-a) z i)\right. \\
& \left.+\frac{e^{2 \sqrt{\pi}(k-a) z i}}{\left(2 \sqrt{\pi}(k-a) z e^{\frac{\pi i}{2}}\right)^{1-s}} \Gamma(1-s, 2 \sqrt{\pi}(k-a) z i)\right) \\
& =\frac{1}{z^{s-1} \sqrt{\pi}} \sum_{k=1}^{\infty}\left(\frac{e^{-2 \sqrt{\pi}(k+a-1) z i}}{\left(2 \sqrt{\pi}(k+a-1) z e^{-\frac{\pi i}{2}}\right)^{1-s}} \Gamma(1-s,-2 \sqrt{\pi}(k+a-1) z i)\right.
\end{aligned}
$$




$$
\begin{array}{r}
\left.+\frac{e^{2 \sqrt{\pi}(k-a) z i}}{\left(2 \sqrt{\pi}(k-a) z e^{\frac{\pi i}{2}}\right)^{1-s}} \Gamma(1-s, 2 \sqrt{\pi}(k-a) z i)\right) \\
+\frac{i}{2 z^{s} \pi} \sum_{k=1}^{\infty}\left(\frac{1}{k-a}-\frac{1}{k+a-1}\right)+\frac{1}{z^{s}} l_{0}(a),
\end{array}
$$

after simplification, which, by writing $\sqrt{\pi} z$ by $z$ becomes

$$
\begin{aligned}
\sum_{k=1}^{\infty} \frac{e^{2 \pi i k a}}{(k+z)^{s}}=\frac{1}{z^{s-1}} \sum_{k=1}^{\infty}\left(\frac{e^{-2 \pi(k+a-1) z i}}{\left(2 \pi(k+a-1) z e^{-\frac{\pi i}{2}}\right)^{1-s}} \Gamma(1-s,-2 \pi(k+a-1) z i)\right. \\
\left.+\frac{e^{2 \pi(k-a) z i}}{\left(2 \pi(k-a) z e^{\frac{\pi i}{2}}\right)^{1-s}} \Gamma(1-s, 2 \pi(k-a) z i)\right)-\frac{1}{2 z^{s}} .
\end{aligned}
$$

Thus we have arrived at the incomplete gamma series for $\phi(a, s, z)$ :

$$
\begin{array}{r}
\phi(a, s, z)=\frac{1}{z^{s-1}} \sum_{k=1}^{\infty}\left(\frac{e^{-2 \pi(k+a-1) z i}}{\left(2 \pi(k+a-1) z e^{-\frac{\pi i}{2}}\right)^{1-s}} \Gamma(1-s,-2 \pi(k+a-1) z i)\right. \\
\left.+\frac{e^{2 \pi(k-a) z i}}{\left(2 \pi(k-a) z e^{\frac{\pi i}{2}}\right)^{1-s}} \Gamma(1-s, 2 \pi(k-a) z i)\right)+\frac{1}{2 z^{s}} .
\end{array}
$$

The deduction, on the basis of the Fourier series for the Dirac delta function, of the following functional equation (2.18) can be found in [1], [11] :

$$
\begin{aligned}
\phi(a, s, z) & =\frac{\Gamma(1-s)}{z^{s-1}} \sum_{k=1}^{\infty}\left(\frac{e^{-2 \pi(k+a-1) z i}}{\left(2 \pi(k+a-1) z e^{-\frac{\pi i}{2}}\right)^{1-s}}+\frac{e^{2 \pi(k-a) z i}}{\left(2 \pi(k-a) z e^{\frac{\pi i}{2}}\right)^{1-s}}\right) \\
& =\frac{\Gamma(1-s)}{(2 \pi)^{1-s}}\left(e^{\frac{1-s}{2} \pi i-2 \pi a z i} \phi(-z, 1-s, a)+e^{-\frac{1-s}{2} \pi i+2 \pi(1-a) z i} \phi(z, 1-s, 1-a)\right)
\end{aligned}
$$

$0<z, a<1$.

\section{Johnson's formula}

In this section we shall first state a very special case of (2.3) with $I=1$ and then deduce Proposition from it.

Corollary. Suppose that two Dirichlet series $\varphi(s)=\sum_{k=1}^{\infty} \frac{\alpha_{k}}{\lambda_{k}^{s}}$ and $\psi(s)=\sum_{k=1}^{\infty} \frac{\beta_{k}}{\mu_{k}^{s}}$ satisfy the functional equation of Hecke type :

$$
\chi(s)=\left\{\begin{array}{l}
\Gamma(s) \varphi(s), \quad \text { if } \Re(s)>\sigma_{\varphi}, \\
\Gamma(r-s) \psi(r-s), \quad \text { if } \Re(s)<\left(r-\sigma_{\psi}\right) .
\end{array}\right.
$$


Then we have

$$
\begin{aligned}
& \sum_{k=1}^{\infty} \frac{\alpha_{k}}{\lambda_{k}^{s}} H_{1,2}^{2,0}\left(\begin{array}{l|c}
z \lambda_{k} & \begin{array}{c}
(b, B) \\
(s, 1),(a, A)
\end{array}
\end{array}\right)=\sum_{k=1}^{\infty} \frac{\beta_{k}}{\mu_{k}^{r-s}} H_{1,2}^{1,1}\left(\frac{\mu_{k}}{z} \mid \begin{array}{c}
(1-a, A) \\
(r-s, 1),(1-b, B)
\end{array}\right) \\
& +\sum_{k=1}^{L} \operatorname{Res}\left(\frac{\Gamma(a-A s+A w)}{\Gamma(b-B s+B w)} \chi(w) z^{s-w}, w=s_{k}\right)
\end{aligned}
$$

which with $a=0, b=s, B=1$ reads

$$
\begin{aligned}
\sum_{k=1}^{\infty} \frac{\alpha_{k}}{\lambda_{k}^{s}} H_{1,2}^{2,0}\left(z \lambda_{k} \mid \begin{array}{c}
- \\
(0, A)
\end{array}\right)= & \sum_{k=1}^{\infty} \frac{\beta_{k}}{\mu_{k}^{r-s}} H_{1,2}^{1,1}\left(\frac{\mu_{k}}{z} \mid \begin{array}{c}
(1, A) \\
(r-s, 1),(1-s, 1)
\end{array}\right) \\
& +\sum_{k=1}^{L} \operatorname{Res}\left(\frac{\Gamma(-A s+A w)}{\Gamma(w)} \chi(w) z^{s-w}, w=s_{k}\right) .
\end{aligned}
$$

Recalling the formula ([16, p.11, (1.7.8)])

$$
H_{1,2}^{1,1}\left(\begin{array}{l|c}
z & (1-a, A) \\
(c, 1),(1-b, B)
\end{array}\right)=z^{c}{ }_{1} \Psi_{1}\left(\begin{array}{r}
(a+A c, A) \\
(b+B c, B)
\end{array} ;-z\right),
$$

where ${ }_{1} \Psi_{1}$ is the Psi series defined by

$$
{ }_{1} \Psi_{1}\left(\begin{array}{c}
(a, A) \\
(b, B)
\end{array} ; z\right)=\sum_{k=0}^{\infty} \frac{\Gamma(a+A k)}{\Gamma(b+B k)} \frac{z^{k}}{k !},
$$

we deduce from (3.2) that

$$
\begin{aligned}
\frac{1}{A} \sum_{k=1}^{\infty} \frac{\alpha_{k}}{\lambda_{k}^{s}} e^{-\sqrt[A]{z \lambda_{k}}}= & z^{s-r} \sum_{k=1}^{\infty} \beta_{k 1} \Psi_{1}\left(\begin{array}{c}
(A(r-s), A) \\
(r, 1)
\end{array} ;-\frac{\mu_{k}}{z}\right) \\
& +\sum_{k=1}^{L} \operatorname{Res}\left(\frac{\Gamma(-A s+A w)}{\Gamma(w)} \chi(w) z^{s-w}, w=s_{k}\right)
\end{aligned}
$$

which is Bochner's formula ([3]).

Proposition (Johnson). ([8]) The generalized Hurwitz-Lerch zeta-function admits the expansion

$$
\Phi_{A}(z, s, a)=\sum_{r=0}^{\infty} \zeta(s-A r, a) \frac{\left(-z^{A}\right)^{r}}{r !}+\frac{\Gamma\left(\frac{1-s}{A}\right)}{A} z^{s-1},
$$

the series on the right being convergent at the origin.

Proof. We apply Corollary to the following two functional equations of Hecke type. For $0<a<1$, we have

$$
\begin{aligned}
\Gamma(s) & \left(\frac{1}{\pi^{s}} \zeta(2 s, a)+\frac{1}{\pi^{s}} \zeta(2 s, 1-a)\right) \\
= & \Gamma\left(\frac{1}{2}-s\right)\left(\frac{1}{\pi^{\frac{1}{2}-s}} l_{1-2 s}(a)+\frac{1}{\pi^{\frac{1}{2}-s}} l_{1-2 s}(-a)\right)
\end{aligned}
$$


with a simple pole at $s_{1}=\frac{1}{2}$, and

$$
\begin{aligned}
\Gamma(s) & \left(\frac{1}{\pi^{s-\frac{1}{2}}} \zeta(2 s-1, a)-\frac{1}{\pi^{s-\frac{1}{2}}} \zeta(2 s-1,1-a)\right) \\
= & \Gamma\left(\frac{3}{2}-s\right)\left(-\frac{i}{\pi^{1-s}} l_{2-2 s}(a)+\frac{i}{\pi^{1-s}} l_{2-2 s}(-a)\right)
\end{aligned}
$$

without poles.

By Corollary, (3.3) yields

$$
\begin{aligned}
& \frac{1}{A} \sum_{k=1}^{\infty} \frac{e^{-\sqrt[A]{z \pi(k+a-1)^{2}}}}{\pi^{s}(k+a-1)^{2 s}}+\frac{1}{A} \sum_{k=1}^{\infty} \frac{e^{-\sqrt[A]{z \pi(k-a)^{2}}}}{\pi^{s}(k-a)^{2 s}} \\
& =z^{s-\frac{1}{2}} \sum_{k=1}^{\infty}\left(e^{2 \pi i k a}+e^{-2 \pi i k a}\right){ }_{1} \Psi_{1}\left(\begin{array}{c}
\left(A\left(\frac{1}{2}-s\right), A\right) \\
\left(\frac{1}{2}, 1\right)
\end{array} ;-\frac{\pi k^{2}}{z}\right)+\frac{\Gamma\left(A\left(\frac{1}{2}-s\right)\right)}{\Gamma\left(\frac{1}{2}\right)} z^{s-\frac{1}{2}}
\end{aligned}
$$

By making change of variables $s \leftarrow \frac{1}{2} s, z \leftarrow \frac{z}{\pi}$, we obtain

$$
\begin{aligned}
& \frac{1}{A} \sum_{k=1}^{\infty} \frac{e^{-\sqrt[A]{z(k+a-1)^{2}}}}{(k+a-1)^{s}}+\frac{1}{A} \sum_{k=1}^{\infty} \frac{e^{-\sqrt[A]{z(k-a)^{2}}}}{(k-a)^{S}} \\
& =\pi^{\frac{1}{2}} z^{\frac{s-1}{2}} \sum_{k=1}^{\infty}\left(e^{2 \pi i k a}+e^{-2 \pi i k a}\right){ }_{1} \Psi_{1}\left(\begin{array}{c}
\left(A \frac{1-s}{2}, A\right) \\
\left(\frac{1}{2}, 1\right)
\end{array} ;-\frac{\pi^{2} k^{2}}{z}\right)+\Gamma\left(A \frac{1-s}{2}\right) z^{\frac{s-1}{2}}
\end{aligned}
$$

In the same way, (3.4) yields

$$
\begin{aligned}
& \frac{1}{A} \sum_{k=1}^{\infty} \frac{e^{-\sqrt[A]{z \pi(k+a-1)^{2}}}}{\pi^{s}(k+a-1)^{2 s-1}}-\frac{1}{A} \sum_{k=1}^{\infty} \frac{e^{-\sqrt[A]{z \pi(k-a)^{2}}}}{\pi^{s}(k-a)^{2 s-1}} \\
& =z^{s-\frac{3}{2}} \sum_{k=1}^{\infty}\left(-i e^{2 \pi i k a}+i e^{-2 \pi i k a}\right){ }_{1} \Psi_{1}\left(\begin{array}{c}
\left(A\left(\frac{3}{2}-s\right), A\right) \\
\left(\frac{3}{2}, 1\right)
\end{array} ;-\frac{\pi k^{2}}{z}\right) .
\end{aligned}
$$

By making change of variables $s \leftarrow \frac{1}{2} s+\frac{1}{2}, z \leftarrow \frac{z}{\pi}$, we obtain

$$
\begin{aligned}
& \frac{1}{A} \sum_{k=1}^{\infty} \frac{e^{-\sqrt[A]{z(k+a-1)^{2}}}}{(k+a-1)^{s}}-\frac{1}{A} \sum_{k=1}^{\infty} \frac{e^{-\sqrt[A]{z(k-a)^{2}}}}{(k-a)^{s}} \\
& =\pi^{\frac{3}{2}} z^{\frac{s-2}{2}} \sum_{k=1}^{\infty} i k\left(-e^{2 \pi i k a}+e^{-2 \pi i k a}\right)_{1} \Psi_{1}\left(\begin{array}{c}
\left(A \frac{2-s}{2}, A\right) \\
\left(\frac{3}{2}, 1\right)
\end{array} ;-\frac{\pi^{2} k^{2}}{z}\right) .
\end{aligned}
$$

Combining the above two formulas, we conclude that

$$
\begin{aligned}
& \frac{1}{A} \sum_{k=1}^{\infty} \frac{e^{-\sqrt[A]{z(k+a-1)^{2}}}}{(k+a-1)^{s}} \\
& =\frac{\pi^{\frac{1}{2}}}{2} z^{\frac{s-1}{2}} \sum_{k=1}^{\infty}\left(e^{2 \pi i k a}+e^{-2 \pi i k a}\right)_{1} \Psi_{1}\left(\begin{array}{c}
\left(A \frac{1-s}{2}, A\right) \\
\left(\frac{1}{2}, 1\right)
\end{array}-\frac{\pi^{2} k^{2}}{z}\right)
\end{aligned}
$$




$$
\begin{aligned}
& +\frac{\pi^{\frac{3}{2}}}{2} z^{\frac{s-2}{2}} \sum_{k=1}^{\infty} i k\left(-e^{2 \pi i k a}+e^{-2 \pi i k a}\right)_{1} \Psi_{1}\left(\begin{array}{c}
\left(A \frac{2-s}{2}, A\right) \\
\left(\frac{3}{2}, 1\right)
\end{array} ;-\frac{\pi^{2} k^{2}}{z}\right) \\
& +\frac{\Gamma\left(A \frac{1-s}{2}\right)}{2} z^{\frac{s-1}{2}}
\end{aligned}
$$

which, after the substitution $A \leftarrow \frac{2}{A}, z \leftarrow z^{2}$, gives rise to

$$
\begin{aligned}
& \sum_{k=1}^{\infty} \frac{e^{-z^{A}(k+a-1)^{A}}}{(k+a-1)^{s}}=\frac{\pi^{\frac{1}{2}}}{A} z^{s-1} \sum_{k=1}^{\infty}\left(e^{2 \pi i k a}+e^{-2 \pi i k a}\right){ }_{1} \Psi_{1}\left(\begin{array}{c}
\left(\frac{1-s}{A}, \frac{2}{A}\right) \\
\left(\frac{1}{2}, 1\right)
\end{array} ;-\frac{\pi^{2} k^{2}}{z^{2}}\right) \\
& +\frac{\pi^{\frac{3}{2}}}{A} z^{\frac{s-2}{A}} \sum_{k=1}^{\infty} i k\left(-e^{2 \pi i k a}+e^{-2 \pi i k a}\right){ }_{1} \Psi_{1}\left(\begin{array}{c}
\left(\frac{2-s}{A}, \frac{2}{A}\right) \\
\left(\frac{3}{2}, 1\right)
\end{array} ;-\frac{\pi^{2} k^{2}}{z^{2}}\right) \\
& +\frac{\Gamma\left(\frac{1-s}{A}\right)}{A} z^{s-1} \\
& =\frac{1}{A} z^{s-1} \sum_{k=1}^{\infty}\left\{e^{2 \pi i k a}{ }_{1} \Psi_{0}\left(\begin{array}{c}
\left(\frac{1-s}{A}, \frac{1}{A}\right) \\
-
\end{array}-\frac{2 \pi i k}{z}\right)\right. \\
& \left.+e^{-2 \pi i k a}{ }_{1} \Psi_{0}\left(\begin{array}{c}
\left(\frac{1-s}{A}, \frac{1}{A}\right) \\
-
\end{array} ; \frac{2 \pi i k}{z}\right)\right\}+\frac{\Gamma\left(\frac{1-s}{A}\right)}{A} z^{s-1},
\end{aligned}
$$

where ${ }_{1} \Psi_{0}$ is another Psi series defined by

$$
{ }_{1} \Psi_{0}\left(\begin{array}{c}
(a, A) \\
-
\end{array} ; z\right)=\sum_{k=0}^{\infty} \Gamma(a+A k) \frac{z^{k}}{k !} .
$$

Note that after analytic continuation we have

$$
{ }_{1} \Psi_{0}\left(\begin{array}{c}
(a, A) \\
-
\end{array} ;-z\right)=\frac{1}{A z^{\frac{a}{A}}} 1 \Psi_{0}\left(\begin{array}{c}
\left(\frac{a}{A}, \frac{1}{A}\right) \\
-
\end{array} ;-\frac{1}{z^{\frac{1}{A}}}\right),
$$

and therefore,

$$
\begin{aligned}
& \sum_{k=1}^{\infty} \frac{e^{-z^{A}(k+a-1)^{A}}}{(k+a-1)^{s}} \\
& =z^{s-1} \sum_{k=1}^{\infty}\left\{e^{2 \pi i k a} \frac{z^{1-s}}{(2 \pi i k)^{1-s}} \Psi_{0}\left(\begin{array}{c}
(1-s, A) \\
-
\end{array} ;-\frac{z^{A}}{(2 \pi i k)^{A}}\right)\right. \\
& \left.+e^{-2 \pi i k a} \frac{z^{1-s}}{(-2 \pi i k)^{1-s} 1} \Psi_{0}\left(\begin{array}{c}
(1-s, A) \\
-
\end{array} ;-\frac{z^{A}}{(-2 \pi i k)^{A}}\right)\right\}+\frac{\Gamma\left(\frac{1-s}{A}\right)}{A} z^{s-1} \\
& =\sum_{k=1}^{\infty}\left\{\frac{e^{2 \pi i k a}}{(2 \pi i k)^{1-s}} \sum_{r=0}^{\infty} \frac{\Gamma(1-s+A r)}{r !}\left(-\frac{z^{A}}{(2 \pi i k)^{A}}\right)^{r}\right. \\
& \left.+\frac{e^{-2 \pi i k a}}{(-2 \pi i k)^{1-s}} \sum_{r=0}^{\infty} \frac{\Gamma(1-s+A r)}{r !}\left(-\frac{z^{A}}{(-2 \pi i k)^{A}}\right)^{r}\right\}+\frac{\Gamma\left(\frac{1-s}{A}\right)}{A} z^{s-1} \\
& =\sum_{r=0}^{\infty} \frac{\Gamma(1-s+A r)}{(2 \pi)^{1-s+A r}} \frac{\left(-z^{A}\right)^{r}}{r !}\left(e^{-\frac{1-s+A r}{2} \pi i} \sum_{k=1}^{\infty} \frac{e^{2 \pi i k a}}{k^{1-s+A r}}+e^{\frac{1-s+A r}{2} \pi i} \sum_{k=1}^{\infty} \frac{e^{-2 \pi i k a}}{k^{1-s+A r}}\right) \\
& +\frac{\Gamma\left(\frac{1-s}{A}\right)}{A} z^{s-1}
\end{aligned}
$$




$$
\begin{aligned}
= & \sum_{r=0}^{\infty} \frac{\Gamma(1-s+A r)}{(2 \pi)^{1-s+A r}} \frac{\left(-z^{A}\right)^{r}}{r !}\left(e^{-\frac{1-s+A r}{2} \pi i} \ell_{1-s+A r}(a)+e^{\frac{1-s+A r}{2} \pi i} \ell_{1-s+A r}(-a)\right) \\
& +\frac{\Gamma\left(\frac{1-s}{A}\right)}{A} z^{s-1} \\
= & \sum_{r=0}^{\infty} \zeta(s-A r, a) \frac{\left(-z^{A}\right)^{r}}{r !}+\frac{\Gamma\left(\frac{1-s}{A}\right)}{A} z^{s-1} .
\end{aligned}
$$

\section{Concluding Remarks}

(i) As is mentioned in Introduction, the Taylor expansion for $\phi(z, s, a)-\Gamma(1-s)(-2 \pi i z)^{s-1}$ was first obtained by Erdélyi et al. ([5, 181, p.49]):

$$
\phi(z, s, a)-\Gamma(1-s)(-2 \pi i z)^{s-1}=\sum_{k=0}^{\infty} \frac{1}{k !} \zeta(s-k, a)(-2 \pi i z)^{k}, \quad(|z|<1),
$$

which was referred to by Johnson as an important result and has been generalized as in Proposition above and by Elizalde [4, (1.31), p.63] as an exact known result. Johnson's formula gives Elizalde's conjectural formula [4, (1.28), p.63].

If we turn our attention to the case $a=1$ and $\zeta(s)$ squared, then the corresponding problem, i. e. the study on $\sum \frac{d(n)}{n^{s}} e^{-z n^{A}}$, first stated by Hardy ([7], footnote, p.7), was studied in more greater detail by Walfisz [19], [20] and by the first author [9].

Walfisz considered the following generalization of Piltz's divisor problem. Let $K$ be an algebraic number field of degree $k$ over $\mathbb{Q}$ with Dedekind zeta function $\zeta_{K}(s)$. Let $Z(s)$ denote the generating series for the $m$-fold divisor function $F(n)$ :

$$
Z(s)=\zeta_{K}(s)^{m}=\sum_{n=1}^{\infty} \frac{F(n)}{n^{s}},
$$

$m$ being a natural number. Then the Piltz divisor problem in $K$ asks for finding a series representation with Bessel function coefficients as well as an asymptotic formula for the summatory function $\sum_{n \leq x} F(n)$. Writing $\kappa=m k$ and

$$
\Psi(z)=\sum_{n=1}^{\infty} \frac{F(n)}{n^{\frac{1}{2}-\frac{1}{2 \kappa}}} e^{-\sqrt[\kappa]{n} z}
$$

Walfisz proved the formula ([20, (4.6), p.163])

$$
\Psi(z)=R(z)+\Phi(z)
$$

where

$$
\Phi(z)=\sum_{n>\frac{\kappa+1}{2}} \frac{(-z)^{n}}{n !} Z\left(\frac{1}{2}+\frac{1}{2 \kappa}-\frac{n}{\kappa}\right)
$$

is a branch of an analytic function regular at the origin and $R(z)$ is the singular part.

The first author considered the product of $\tau$ Dedekind zeta-functions associated to $\tau$ algebraic number fields $K_{j}, 1 \leq j \leq \tau$ :

$$
Z^{*}(s)=\prod_{j=1}^{\tau} \zeta_{K_{j}}(s)=\sum_{n=1}^{\infty} \frac{D(n)}{n^{s}}
$$


and studied Hardy's original problem in the most general setting:

$$
F(z)=F_{s, A}(z)=\sum_{n=1}^{\infty} \frac{D(n)}{n^{s}} e^{-z n^{A}},
$$

for which he deduced the Taylor expansion $\left(\left[9,\left(8^{\prime}\right)\right.\right.$, p.215]).

Thus, Hardy's statement that $\sum_{n=1}^{\infty} \frac{d(n)}{n^{s}} e^{-z n^{A}}$ may be expressed as a main term plus an integral function should be modified as a main term plus an analytic function regular at the origin.

As with Hardy for whom the Taylor expansion was just a starting point for establishing his most famous omega results, so with Walfisz, who, decomposing the $\Psi(z)$-function into a finite sum of $M(z)$-functions, expressed $M(z)$-function as infinite sum of $L(z)$-functions and then studied the singularities as in Hardy [7] to establish the omega results. A novel point is that he appealed to the theory of linear differential equation satisfied by

$$
L(z)=\sum_{k=0}^{\infty} \frac{\Gamma^{\kappa}\left(\frac{1}{2}-\frac{1}{2 \kappa}+\frac{k}{\kappa}\right)}{\Gamma(k+1)} z^{k} .
$$

The first author went further from the Taylor expansion to decompose $F(z)$ into a finite sum of $M_{\mu}(z)$-functions, which he further expressed as an infinite sum of $L_{\mu}(z)$-functions, where

$$
L_{\mu}(z)=\sum_{\substack{k=2 \nu \\ k \equiv \mu}} \frac{\Gamma(1-\alpha+\beta k)^{\nu}}{\Gamma(k+1)} z^{k},
$$

and where $\nu$ signifies the sum of degrees of $K_{j}$ 's over $\mathbb{Q}$. The main result is the expression of $L_{\mu}(z)$-functions as Meijer's $G$-functions, a genesis of the recent researches by us ([13], [18] etc.).

Thus we see that Johnson's formula is the starting point of further research and can be proved most easily by residue calculus. However, if we appeal to this, we will lose the most important point to the effect that the formula is one obtained by applying the functional equation twice.

Moreover, in the study of $L(z)$-functions, one can see an interaction of three different mathematical disciplines, number theory, differential equations and special functions.

(ii) Examples of the class of zeta-functions satisfying the ramified functional equation are hardly exhausted by what is given in this note. It surely includes the Estermann zetafunction studied by M. Jutila, the product of the Hurwitz zeta-functions as referred to in [10], or even the perturbed type series

$$
\sum_{n=0}^{\infty} \frac{d(n)}{(n+\alpha)^{s}} e^{-z n^{A}}
$$

etc. We shall return to these examples elsewhere.

(iii) Also, some of the zeta-regularization problems may have already been solved in the study of number-theoretic problems as we have seen above in the case of the Dirichlet divisor problem and its generalization. We hope to return to elucidation of this area in the future.

Acknowledgements. Part of this work was carried out when the first two authors were staying at Number Theory Institute of North West University, Xi'an, PRC. They express hearty thanks to the Institute and the University for the excellent working conditions and hosipitality of the staffs. 


\section{References}

[1] R. Balasubramanian, S. Kanemitsu and H. Tsukada, Contributions to the theory of the Lerch zeta-function, to appear

[2] S. Bochner, Some properties of modular relations, Ann. of Math. 53 (1951), 332-363.

[3] S. Bochner, On Riemann's Functional Equation with Multiple Gamma Factors, Ann. of Math. 67 (1958).

[4] E. Elizalde, S. D. Odnitsov, A. Romeo, A. A. Bytsenko and S. Zerbini, Zeta regularization techniques with applications, World Scientific, Singapore-New Jersey-London-Hong Kong, 1994.

[5] A. Erdelyi, Higher Transcendental Functions I, McGraw-Hill, New York, 1953.

[6] C. Fox, The $G$ and $H$ functions as symmetric Fourier kernels, Trans. Amer. Math. Soc. 98 (1961), 395-429.

[7] G. H. Hardy, On Dirichlet's divisor problem, Proc. London Math. Soc. (2) 15 (1916), $1-25$.

[8] B. R. Johnson, Generalized Lerch zeta function, Pacific J. Math. 53 (1974), 191-193.

[9] S. Kanemitsu, On a general divisor problem, Mem. Fac. Sci. Kyushu Univ. Ser. A 32 (1978), 211-221.

[10] S. Kanemitsu and W. Recknagel, Special functions in number theory, Kyoyo Ronshu (Kinki University) Vol. 5, No. 5 and Vol. 6, No.1, 89-109.

[11] S. Kanemitsu, Y. Tanigawa, H. Tsukada and M. Yoshimoto, Contributions to the theory of the Hurwitz zeta-function, preprint.

[12] S. Kanemitsu, Y. Tanigawa, H. Tsukada and M. Yoshimoto, Some aspects of the modular relation, preprint.

[13] S. Kanemitsu, Y. Tanigawa, H. Tsukada and M. Yoshimoto, Contributions to the theory of zeta-functions: the modular relation supremacy, in preparation.

[14] S. Kanemitsu, Y. Tanigawa and M. Yoshimoto, Ramanujan's formula and modular forms, Number Theoretic Method-Future Trends, (ed. S. Kanemitsu and C. Jia) Kluwer Acakdemic Publisher, Dordrecht (2003), 159-212.

[15] A. Laurinčikas and R. Garunkštis, The Lerch zeta-function, Kluwer Academic Publ., Dordrecht-Boston-London 2002.

[16] A. M. Mathai and R. K. Saxena, The H-fucntion with applications in statistics and other disciplines, Wiley Eastern, New Delhi 1978.

[17] A. P. Prudnikov, Yu.A. Bychkov and O.I. Marichev, Integrals and series, supplementary chapters, Izd. Nauka, Moscow 1986.

[18] H. Tsukada, A general functional equation in analytic number theory, submitted.

[19] A. Walfisz, Über die summatorischen Funktionen einiger Dirichletscher Reihen, Inaugural-Dissertation, Gottingen 1922, 58 pages. 
[20] A. Walfisz, Über das Piltzsche Teilerproblem in algebraischen Zahlkörpern, Math. Z. 22 (1925), 153-188.

Shigeru Kanemitsu

Kinki University School of Humanity-Oriented Science and Engineering,

Iizuka, Fukuoka, 820-8555, Japan

e-mail: kanemitu@fuk.kindai.ac.jp

Yoshio Tanigawa

Graduate School of Mathematics, Nagoya University,

Nagoya, 464-8602, Japan

e-mail: tanigawa@math.nagoya-u.ac.jp

Haruo Tsukada

Kinki University School of Humanity-Oriented Science and Engineering,

Iizuka, Fukuoka, 820-8555, Japan

e-mail: tsukada@fuk.kindai.ac.jp 